\title{
Incidence of airflow limitation in subjects 65-100 years of age
}

\author{
Johannes A. Luoto ${ }^{1}$, Sölve Elmståhl ${ }^{1}$, Per Wollmer ${ }^{2}$ and Mats Pihlsgård ${ }^{1}$ \\ Affiliations: \\ ${ }^{1}$ Dept of Health Sciences, Division of Geriatric Medicine, Scania University Hospital, Lund University, Malmö, \\ Sweden. \\ ${ }^{2}$ Clinical Physiology and Nuclear Medicine Unit, Dept of Translational Medicine, Scania University Hospital, \\ Lund University, Malmö, Sweden.
}

\section{Correspondence:}

Johannes A. Luoto, Dept of Health Sciences, Division of Geriatric Medicine, Jan Waldenströms gata 35, 214 21, Malmö, Sweden.

E-mail: johannes.luotolamed.lu.se

ABSTRACT The true incidence of chronic obstructive pulmonary disease is largely unknown, because the few longitudinal studies performed have used diagnostic criteria no longer recommended by either the European Respiratory Society or the American Thoracic Society (ATS).

We studied the incidence and significance of airflow limitation in a population-based geriatric sample using both an age-dependent predicted lower limit of normal (LLN) value and a fixed-ratio spirometric criterion.

Out of 2025 subjects with acceptable spirometry at baseline, 984 subjects aged 65-100 years completed a 6-year follow-up visit. Smoking habits were registered at baseline. Exclusion criteria were non-acceptable spirometry performance according to ATS criteria and inability to communicate. Airflow limitation was defined both according to forced expiratory volume in $1 \mathrm{~s}(\mathrm{FEV} 1) /$ forced vital capacity ratio $<0.7$ and $<$ LLN.

The incidence of airflow limitation per 1000 person-years was 28.2 using a fixed ratio and 11.7 with LLN, corresponding to a 1.41-fold higher incidence rate using a fixed ratio. The incidence increased dramatically with age when using a fixed ratio, but less so when using LLN. In addition, a sex effect was observed with the LLN criterion. LLN airflow limitation was associated with increased 5-year mortality. Presence of fixed-ratio airflow limitation in individuals classified by LLN as non-obstructive was not associated with increased mortality.

@ERSpublications

Female sex may be a risk factor for developing chronic obstructive pulmonary disease http://ow.ly/TdgEd

For editorial comment see Eur Respir J 2016; 47: 379-381 [DOI: 10.1183/13993003.01806-2015].

Received: April 222015 | Accepted after revision: Sept 242015 | First published online: Dec 172015

Support statement: S. Elmståhl, J.A. Luoto and M. Pihlsgård all received grants from the Swedish Ministry of Health and Social Affairs, grants from Skåne county and grants from the Swedish Research Council (grant no. 2013-8604), during the conduct of the study. Funding information for this study has been deposited with FundRef.

Conflict of interest: Disclosures can be found alongside the online version of this article at erj.ersjournals.com

Copyright CERS 2016. ERJ Open articles are open access and distributed under the terms of the Creative Commons Attribution Non-Commercial Licence 4.0. 


\section{Introduction}

The incidence of chronic obstructive pulmonary disease (COPD) using current diagnostic criteria is largely unknown, particularly in the oldest age groups [1].

A literature review found few recent studies (published 2000-2010) covering the incidence of COPD. The reported incidences vary widely between studied populations and depend on the diagnostic criteria used [1]. Although spirometry-verified airflow limitation is the standard method for diagnosing COPD, only four out of 15 articles in the review used spirometry as a diagnostic method [2-5]. Another five studies published after the year 2000, not mentioned in the review, report spirometry-based incidence [6-10]. Two of these previous studies reports incidence of airflow limitation based on the ratio of age-dependent predicted lower limits of normal (LLN) (forced expiratory volume in $1 \mathrm{~s}$ (FEV1)/forced vital capacity $(\mathrm{FVC})<\mathrm{LLN})[9,10]$ and the others use a fixed ratio $(\mathrm{FEV} 1 / \mathrm{FVC}<0.7)$ that is independent of age.

The discussion on whether to use a fixed ratio or the LLN for defining airflow limitation is ongoing, due to the reported risk of misclassifying elderly individuals as pathologically obstructive when using a fixed ratio [11-15]. Guidelines issued by the Global Initiative for Chronic Obstructive Lung Disease (GOLD) recommend using a fixed ratio of 0.7 [16], while the American Thoracic Society (ATS) and European Respiratory Society (ERS) recommend the use of LLN [17]. The ERS task force has recently strengthened the case for using LLN due to the reported risk of underdiagnosing subjects aged <50 years and overdiagnosing subjects aged $>50$ years, with $75-80 \%$ false positives among subjects aged 80 years when using a fixed ratio [18]. In the light of these facts, new studies on incidence rates and risk factors for incident COPD based on appropriate diagnostic criteria are required.

Smoking and age were significant predictors of airflow limitation in all of the aforementioned studies. We have not found any previously published incidence rates for subjects aged $>80$ years. Two studies also reported a significant dose-response relationship between incidence and estimated smoking quantity $[4,6]$. With regard to sex as a risk factor for developing COPD, the evidence is not clear-cut and seems to depend on the diagnostic criteria used $[4,5]$.

In this study we established the incidence of airflow limitation in a population that includes subjects aged up to 100 years at follow-up, using both the predicted LLN and a fixed-ratio cut-off of 0.7 for diagnosis. We also established the differences in incidence between different age groups, sexes and groups based on smoking habits. Furthermore, we assessed and compared the significance in terms of mortality of being diagnosed with airflow limitation according to the criteria above. The primary hypothesis was that incidence increases with age and is associated with smoking habits but not with sex. The secondary hypothesis was that the incidence has a stronger correlation with age and is generally higher when using the fixed-ratio criterion compared to the LLN criterion.

\section{Materials and methods}

Study description and participation

This study is part of the longitudinal Good Ageing in Skåne study, which in turn is part of the Swedish National Study on Ageing and Care. These studies have been described previously [19-21]. Initially, 5370 subjects (mean age 74.7 years) were invited. Of these, 477 (mean age 80.1 years) were non-eligible. Of the remainder, 1962 (mean age 76.1 years) were non-participants and 2931 (mean age 72.9 years) accepted the invitation. Baseline examinations were undertaken between February 2001 and January 2004, and the follow-up took place $\sim 6$ years later. Age of participants at follow-up was 65-100 years. The baseline assessment included a questionnaire about smoking habits as well as medical examinations performed by a physician and a nurse. The assessments were performed during one or two consecutive days at the research centre or at the subject's home or in sheltered housing. Making house calls was a deliberate strategy to reduce selection bias. Exclusion criteria were twofold: 1) absence of spirometry or unacceptable spirometry performance according to ATS criteria [22] and 2) inability to communicate in Swedish and no available interpreter.

Baseline spirometry was performed on 2336 subjects aged 60-95 years from both rural and urban general populations of the Swedish province of Scania. 2025 subjects performed acceptable spirometry at baseline according to ATS acceptability criteria [22], of which 984 also produced acceptable spirometry at follow-up. The proportion of house calls was $12 \%$ at baseline and $16 \%$ at follow-up. Subjects were classified as regular smokers, intermittent smokers, ex-smokers or never-smokers. A quantification of smoked cigarettes in pack-years was estimated by multiplying the self-reported average number of packs of cigarettes smoked per day by the number of reported years of smoking. Regular smokers and intermittent smokers were merged into one group called current smokers on account of the relatively small number $(n=76)$ of intermittent smokers. Four age groups (60-69 years, 70-79 years, 80-89 years and 90-100 years) were defined. For each participant, we recorded study dropout or death, whichever happened first, with the corresponding date. See figure 1 for an overview of the study. Baseline characteristics at different levels of participation are shown in table 1. 
Dropout, death and missing data among participants

In an attempt to assess whether subjects who did not perform acceptable spirometry at baseline had poorer pulmonary function than those who did, we compared the prevalence of obstruction at follow-up. We tested whether obstruction status at baseline was related to death or dropout in between examinations. To see whether the dropout or death resulted in relatively fitter older groups, we tested whether differences (between those lost to follow-up and those with two spirometries) in age and outcome of walking test at baseline depended on age group. More precisely, we regressed age and outcome of walking test at baseline on a full interaction of loss to follow-up and age group and subsequently conducted suitable contrast tests.

\section{Definitions of airflow limitation and incidence}

We report measured airflow limitation as an estimate of COPD. Airflow limitation is defined as a disproportionate reduction of the maximal airflow $\left(\mathrm{FEV}_{1}\right)$ in relation to the maximal volume (FVC or $\mathrm{VC}$ ) [17]. The applied criteria for airflow limitation in this study are both based on the ratio $r=F_{E V} /(F) V C$. The fixed-ratio criterion is defined as $r<0.7$ [16]. The LLN criterion is defined as $r<$ predicted LLN. We used the 2012 Global Lungs Initiative (GLI) reference equations to calculate the predicted values [23]. Ethnicity was set to Caucasian. Background and more details on these reference equations have been published by the ERS task force [18]. For subjects aged $>95$ years at follow-up we extrapolated the formulas for predicted LLN in order to keep these subjects in the analysis. For obstructive subjects we calculated the severity of obstruction according to GOLD and ATS classifications $[16,17]$.

Incidence is based on two measurements, an initial baseline measurement and a 6-year follow-up measurement. We evaluated the airflow limitation definitions by looking at the association of baseline obstruction with future 5-year mortality rates. To test that reclassification was not mainly caused by natural variability and measurement error, we compared the decline in FEV1 among incident positive and incident negative (obstructive at baseline and non-obstructive at follow-up) subjects. We also compared change in FEV1 between incident positive and non-incident (non-obstructive at baseline and follow-up) to test if reclassification corresponded to a pathological decline. To assess the effect of general health status on incidence, we conducted a separate analysis on subjects not examined at the research centre. The distribution and severity of prevalent and incident obstruction, background variables, dropout patterns and associated survival are displayed in tables 2 and 3 .

\section{Spirometry}

The tests were performed using the Vitalograph 2120 electronic flow volume spirometer using Spirotrac IV software (Vitalograph Ltd, Buckingham, UK). The same spirometer was used when the test was performed at locations other than the research centre. The spirometry, including calibration, was performed according to ATS guidelines [22] at both baseline and the 6-year follow-up with one exception: at baseline subjects did not receive a bronchodilator, whereas at follow-up subjects received $1.0 \mathrm{mg}$ of $\beta_{2}$-receptor agonist terbutaline $10 \mathrm{~min}$ prior to the spirometry. In order to assess crudely to what extent this inconsistency may have influenced the results we conducted two additional experimental incidence analyses. In the first (for the estimation of potential negative effect on incidence rates) we assumed that all incident negative subjects had been non-obstructive at baseline had they inhaled a bronchodilator. In the second (for the estimation of potential positive effect on incidence rates) we assumed a significant increase of FEV1 of $10 \%$ of measured value and no increase in VC after bronchodilation [24].

\section{Statistical analysis}

In order to assess differences in incidence rate between subgroups, we conducted Poisson regression analyses with age, smoking category and sex as covariates. Only main effects were included. The exposure time, i.e. the time between baseline and follow-up, was taken into account by including an appropriate offset variable in the analyses. Incidence rate should be interpreted as expected incidence of airflow limitation per 1000 person-years. 5-year mortality rates were analysed using Cox regression with the minimum of 5 years and time elapsed between baseline and dropout or death as outcome, with censoring corresponding to dropout or still being in the study 5 years after baseline. In addition to obstruction status (non-obstructive, obstructive only with fixed ratio and obstructive with LLN), the model was adjusted for age, smoking, sex, cardiovascular disease and type 2 diabetes. The analysis concerning varying dropout patterns in different age groups was based on regression with Gaussian errors. The analysis of FEV1 decline difference between incident positive and incident negative groups was conducted using Wilcoxon's signed rank test and rank sum test. When crudely comparing the distributions of a categorical outcome in different strata, Chi-squared tests were used. When making comparisons, a p-value $<0.05$ was considered statistically significant. All analyses were performed using SAS 9.4 (SAS Institute Inc., Cary, NC, USA). 


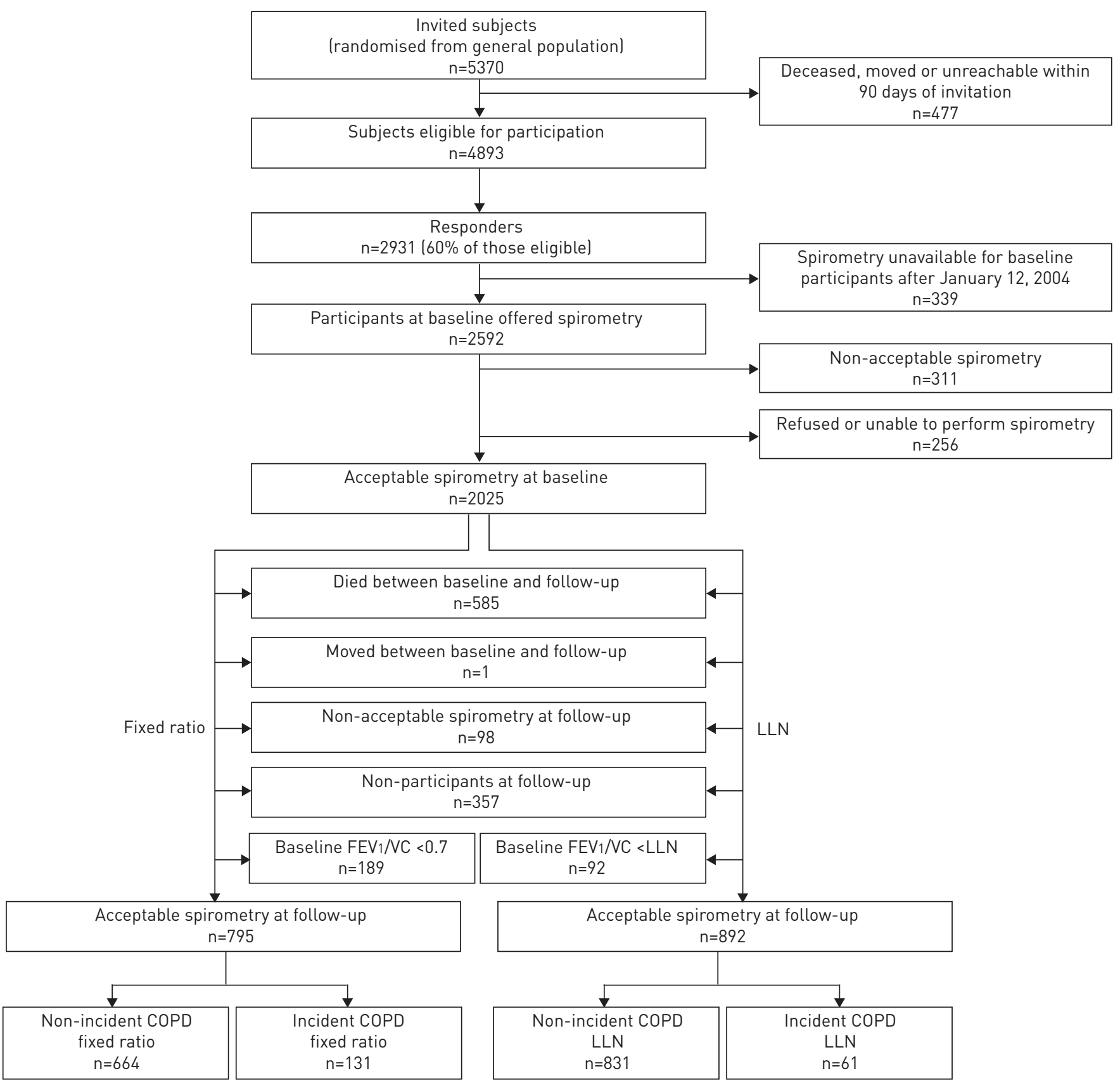

FIGURE 1 Study overview. LLN: lower limit of normal; FEV1: forced expiratory volume in $1 \mathrm{~s}$; VC: vital capacity; COPD: chronic obstructive pulmonary disease.

Ethics

The study was approved by the regional ethics committee of Lund University (2002; registration number LU 744-00) and all participants provided written consent.

\section{Results}

Participants

Of the 311 subjects without acceptable spirometry at baseline, 204 (65.6\%) produced acceptable spirometry at follow-up. The prevalence of obstruction at follow-up did not differ between these and the 984 with spirometry at baseline ( $p=0.34$ (fixed ratio) and $p=0.35$ (LLN)). There was an effect of baseline obstruction on death prior to follow-up ( $\mathrm{p}<0.0001$ for fixed ratio and LLN), but we saw no such effect for dropout $(\mathrm{p}=0.91)$. Dropout or death did seem to result in the older groups being relatively fitter $(\mathrm{p}=0.019$ and $\mathrm{p}=0.0041$ for age and outcome of walking test, respectively). Of the 2025 participants with baseline 
TABLE 1 Baseline characteristics according to study participation

\begin{tabular}{ccccc}
\multicolumn{2}{c}{ Invited } & & & Spirome \\
\cline { 1 - 1 } & Responders & $\begin{array}{c}\text { Acceptable } \\
\text { at baseline }\end{array}$ & $\begin{array}{c}\text { Non-acceptable } \\
\text { at baseline }\end{array}$ & $\begin{array}{c}\text { Acceptable at } \\
\text { baseline/ } \\
\text { non-acceptable } \\
\text { at follow-up }\end{array}$
\end{tabular}

Acceptable at baseline and follow-up

at follow-up

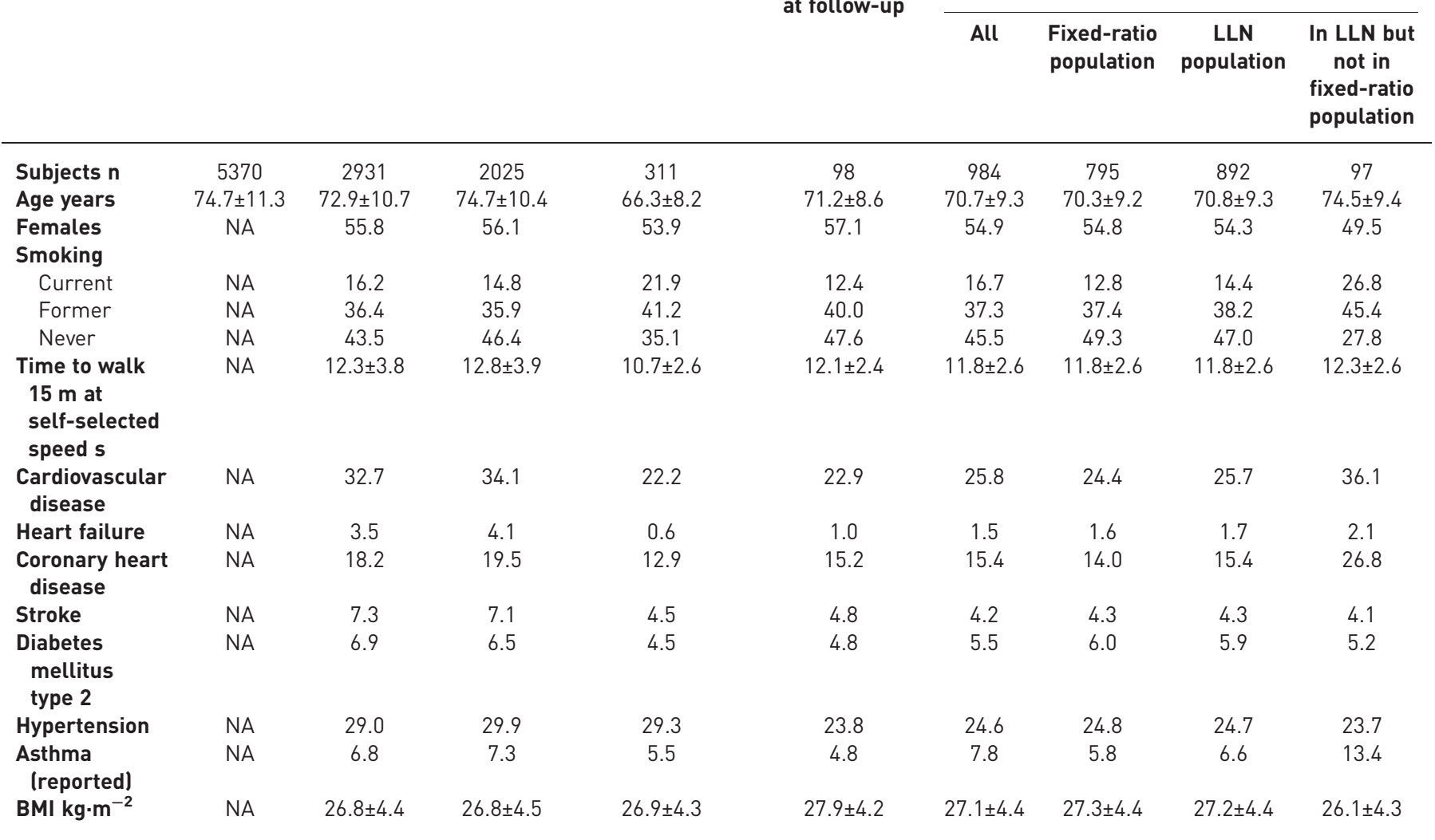

Data are presented as mean \pm SD or $\%$, unless otherwise stated. LLN: lower limit of normal; BMI: body mass index; NA: not applicable.

spirometry, $1137(56.2 \%)$ were tested on one specific spirometer. Of the 311 with non-acceptable baseline spirometries, $273(87.8 \%)$ were tested using this machine. At baseline, 189 and 92 participants were qualified as obstructive by fixed ratio and LLN, respectively. Of these, 68 and 34, respectively, were non-obstructive at follow-up (incident negative).

Significances of criteria and incidences based on r $<$ fixed ratio and $r<L L N$

Compared to being non-obstructive, fixed-ratio obstruction alone was not correlated with the 5-year mortality rate $(\mathrm{p}=0.12)$, whereas being obstructive according to LLN was correlated with increased mortality with a rate ratio of $1.9(\mathrm{p}<0.0001)$. A significant effect on mortality $(\mathrm{p}=0.0062)$ of LLN obstruction relative to obstruction only with fixed ratio was observed (rate ratio=1.6). Regardless of criterion, for the incident positive group there was evidence against symmetry around zero of the decline in FEV1 $(\mathrm{p}<0.0001)$. For the incident negative group, we could not discard symmetry ( $\mathrm{p}=0.053$ (fixed ratio) and $\mathrm{p}=0.73(\mathrm{LLN})$ ). The decline in FEV1 differs between incident positive and incident negative subjects for both fixed ratio and LLN ( $\mathrm{p}<0.0001$ and $\mathrm{p}=0.0002$, respectively) with incidence corresponding to a greater decline. Decline in FEV 1 for the incident positive group was significantly larger for both LLN $(\mathrm{p}=0.0004)$ and fixed ratio $(\mathrm{p}<0.0001)$ than for the non-incident group.

\section{Incidence rates}

Average follow-up time was 5.8 years (fixed ratio) and 5.9 years (LLN), respectively. Incidence rate (crude and adjusted via least-squares means) with corresponding 95\% confidence intervals are displayed in table 4 (fixed ratio) and table 5 (LLN). Least-squares means are predicted population margins assuming balanced data. Figure 2 illustrates the nonlinear age effect on incident airflow limitation for LLN and fixed ratio. Note that we used age as a continuous covariate in figure 2. The crude incidence rate was 1.41 times 
TABLE 2 Characteristics of subjects with acceptable spirometry at baseline according to prevalence and severity of obstruction

\begin{tabular}{|c|c|c|c|c|c|c|c|c|c|c|c|c|c|}
\hline & \multirow{3}{*}{$\begin{array}{c}\mathrm{FEV}_{1} / \mathrm{VC} \\
>0.7\end{array}$} & \multicolumn{5}{|c|}{$\mathrm{FEV}_{1} / \mathrm{VC}<0.7$} & \multirow{3}{*}{$\begin{array}{c}\text { FEV1/VC } \\
>\text { LLN }\end{array}$} & \multicolumn{6}{|c|}{ FEV $1 / V C<L L N$} \\
\hline & & \multirow[t]{2}{*}{ All } & \multicolumn{4}{|c|}{ GOLD stage } & & \multirow[t]{2}{*}{ All } & \multicolumn{5}{|c|}{ ATS/ERS 2005 severity [16] } \\
\hline & & & $\mathbf{I}$ & II & III & IV & & & 1 & 2 & 3 & 4 & 5 \\
\hline \multirow[t]{2}{*}{ Subjects n $(\%)$} & 1489 & 536 & 200 & 231 & 65 & 36 & 1753 & 256 & 103 & 37 & 29 & 47 & 40 \\
\hline & (73.5) & $(26.5)$ & $(9.9)$ & (11.4) & $(3.2)$ & (1.8) & (87.3) & $(12.7)$ & (5.1) & $(1.8)$ & $(1.4)$ & $(2.3)$ & (2.0) \\
\hline \multirow[t]{2}{*}{ Age years } & 73.9 & 77.2 & 75.9 & 77.5 & 77.4 & 81.9 & 74.5 & 75.7 & 72.2 & 76.9 & 80.5 & 75.3 & 80.7 \\
\hline & \pm 10.3 & \pm 10.2 & \pm 10.7 & \pm 9.5 & \pm 11.4 & \pm 7.4 & \pm 10.4 & \pm 10.6 & \pm 10.9 & \pm 9.9 & \pm 7.1 & \pm 11.5 & \pm 8.3 \\
\hline \multicolumn{14}{|l|}{ Smoking } \\
\hline Current & 11.6 & 23.7 & 20.0 & 27.3 & 25.2 & 13.9 & 12.8 & 28.1 & 32.0 & 32.4 & 24.1 & 29.8 & 15.0 \\
\hline Former & 35.6 & 36.8 & 35.0 & 39.0 & 38.5 & 27.8 & 36.1 & 34.8 & 34.0 & 32.4 & 34.5 & 40.4 & 32.5 \\
\hline Never & 50.4 & 35.1 & 42.5 & 30.7 & 21.5 & 50.0 & 48.6 & 31.3 & 32.0 & 29.7 & 37.9 & 17.0 & 42.5 \\
\hline CVD & 31.7 & 40.7 & 31.5 & 47.6 & 44.6 & 38.9 & 33.4 & 37.9 & 29.1 & 51.4 & 41.4 & 42.6 & 40.0 \\
\hline Heart failure & 3.6 & 5.4 & 2.0 & 7.8 & 4.6 & 11.1 & 3.9 & 5.5 & 1.0 & 10.8 & 13.8 & 4.3 & 7.5 \\
\hline $\begin{array}{l}\text { Diabetes mellitus } \\
\text { type } 2\end{array}$ & 6.8 & 5.8 & 5.0 & 6.5 & 6.2 & 5.6 & 6.8 & 4.7 & 3.9 & 8.1 & 0.0 & 6.4 & 5.0 \\
\hline In follow-up & 65.9 & 48.1 & 60.0 & 45.0 & 32.3 & 36.1 & 63.7 & 46.9 & 64.1 & 32.4 & 41.4 & 34.0 & 35.0 \\
\hline $\begin{array}{l}\text { In follow-up with } \\
\text { spirometry }\end{array}$ & 53.4 & 35.5 & 45.5 & 32.0 & 26.2 & 19.4 & 50.9 & 35.9 & 48.5 & 21.6 & 24.1 & 36.2 & 25.0 \\
\hline
\end{tabular}

Data are presented as mean \pm SD or $\%$, unless otherwise stated. Fixed-ratio population: $n=2025$; lower limit of normal (LLN) population: $n=2009$ (data not available for LLN calculation for 16 subjects). FEV1: forced expiratory volume in 1 s; VC: vital capacity; GOLD: Global Initiative for Chronic Obstructive Lung Disease; ATS: American Thoracic Society; ERS: European Respiratory Society; CVD: cardiovascular disease (heart disease or stroke/transient ischaemic attack).

higher with a fixed ratio than with LLN for the entire study population. The crude incidence rate for current smokers compared to never-smokers was 0.30 times higher with a fixed ratio and 0.61 times higher with LLN. The incidence rate of females was 0.14 times higher than that of males by fixed ratio, and 1.02 times higher by LLN.

\section{Analysis of predictors}

The results from the regression analyses are presented in tables 4 and 5 . We focus solely on the results from the multivariate analyses. Regardless of airflow limitation criterion, we saw statistically significant effects (relative to the youngest age group) corresponding to the two intermediate age groups. However, the global age effect was much weaker for LLN; when testing for equality of incidence rate across age groups, we obtained $\mathrm{p}<0.0001$ for fixed ratio and $\mathrm{p}=0.0415$ for LLN ( $\mathrm{p}$-values not shown in tables). Relative to 80-89-year-olds, we observed no significant effect of belonging to the oldest age group ( $\mathrm{p}=0.807$ (fixed ratio) and $\mathrm{p}=0.981$ (LLN)). We observed a significant effect (relative to never-smokers) of being a current smoker for both fixed-ratio and LLN criteria. A multivariate analysis of pack-years as a continuous variable with sex and age as covariates was also conducted, showing a significant relationship between pack-years and the incidence rate of airflow limitation for both the fixed ratio and LLN population ( $\mathrm{p}=0.0173$ (fixed ratio) and $\mathrm{p}=0.009$ (LLN)). We saw a significant sex effect for LLN, which was not present for the fixed-ratio criterion, with females having a higher incidence rate.

\section{Additional incidence analyses}

Place of examination

The incidence rate among subjects examined outside the research centre was 50.2 (95\% CI 22.5-111.7) with fixed ratio and 14.6 (95\% CI 3.7-58.5) with LLN. There was no significant effect of place of examination with neither fixed ratio nor LLN ( $\mathrm{p}=0.15$ and $\mathrm{p}=0.75$, respectively).

Hypothetical bronchodilator effect at baseline

A potential positive effect on incidence rates was calculated: the effect of a $10 \% \mathrm{FEV}_{1}$ increase and no (F)VC increase of measured baseline value led to a 0.30 times higher adjusted incidence rate for fixed ratio (increased to 48.4) and 0.25 times higher for LLN (increased to 17.6). A dramatic effect of smoking was also 
TABLE 3 Characteristics of subjects with acceptable spirometry at both baseline and follow-up according to incidence and severity of incident obstruction

\begin{tabular}{|c|c|c|c|c|c|c|c|c|c|c|c|c|c|}
\hline & \multicolumn{6}{|c|}{ In fixed-ratio incidence analysis } & \multicolumn{7}{|c|}{ In LLN incidence analysis } \\
\hline & \multirow{3}{*}{$\begin{array}{l}\text { Non-incident } \\
\text { fixed ratio }\end{array}$} & \multicolumn{5}{|c|}{ Incident fixed ratio } & \multirow{3}{*}{$\begin{array}{c}\text { Non-incident } \\
\text { LLN }\end{array}$} & \multicolumn{6}{|c|}{ Incident LLN } \\
\hline & & \multirow[t]{2}{*}{ All } & \multicolumn{4}{|c|}{ GOLD stage follow-up \# } & & \multirow[t]{2}{*}{ All } & \multicolumn{5}{|c|}{ ATS/ERS 2005 severity [16] follow-up } \\
\hline & & & I & II & III & IV & & & 1 & 2 & 3 & 4 & 5 \\
\hline Subjects $n(\%)$ & $664(76.9)$ & $131(16.5)$ & 84 (9.7) & 34 (3.9) & $6(0.7)$ & $1(0.1)$ & $831(89.7)$ & $61(6.6)$ & 39 (3.7) & $10(1.1)$ & $7(0.8)$ & $4(0.4)$ & $1(0.1)$ \\
\hline $\begin{array}{l}\text { Females } \\
\text { at baseline }\end{array}$ & 54.4 & 57.3 & 58.3 & 55.9 & 33.3 & 100.0 & 53.1 & 70.5 & 76.9 & 70.0 & 57.1 & 50.0 & 0.0 \\
\hline \multicolumn{14}{|l|}{$\begin{array}{c}\text { Smoking at } \\
\text { baseline }\end{array}$} \\
\hline Current & 12.4 & 15.3 & 11.9 & 26.5 & 16.7 & 0.0 & 13.7 & 23.0 & 15.4 & 30.0 & 28.6 & 75.0 & 0.0 \\
\hline Former & 37.1 & 38.9 & 33.3 & 47.1 & 83.3 & 0.0 & 38.8 & 31.2 & 30.8 & 30.0 & 28.6 & 25.0 & 100.0 \\
\hline Asthma? & 6.5 & 9.2 & 6.0 & 14.7 & 33.3 & 0.0 & 7.5 & 13.1 & 7.7 & 20.0 & 14.3 & 50.0 & 0.0 \\
\hline
\end{tabular}

Data are presented as mean \pm SD or \%, unless otherwise stated. LLN: lower limit of normal; GOLD: Global Initiative for Chronic Obstructive Lung Disease; ATS: American Thoracic Society; ERS: European Respiratory Society; CVD: cardiovascular disease (heart disease or stroke/transient ischaemic attack). " : data not available for 6 subjects; ๆ: at baseline or follow-up.

observed for the fixed-ratio criterion. For LLN, it was associated with a nonsignificant effect of belonging to the 70-79-years age group $(\mathrm{p}=0.08)$. For potential negative effect on incidence rates, the effect of incident-negative becoming non-incident resulted in slightly lower incidence rates for both the fixed ratio (adjusted incidence rate 32.6) and LLN (adjusted incidence rate 13.8) criteria. For LLN, effects of predictors were not altered, for fixed ratio the effect of being a current smoker became nonsignificant $(\mathrm{p}=0.08)$.

\section{Discussion \\ Comparison of diagnoses}

The analysis of 5-year mortality revealed that an obstructive ventilatory pattern at baseline according to the fixed-ratio criterion alone was not associated with an increased risk of all-cause mortality compared to a non-obstructive pattern according to both criteria. In contrast, the effect of LLN-obstruction was strong, corresponding to nearly a doubling of the mortality rate. This is consistent with previous findings and suggested once again that in an elderly population the fixed-ratio criterion was not entirely appropriate for diagnosing airflow limitation and that the LLN provided a better alternative in terms of a lower risk of false-positivity without missing subjects at increased risk of mortality $[25,26]$.

\section{Brief synopsis of key findings regarding incidence}

Regardless of criterion, the incident positive group showed a greater decline in FEV1 compared to the incident negative and non-incident groups. This supports the idea that reclassification was not due to measurement error and natural variability. The overall crude incidence rate was 1.41 times higher using the fixed ratio criterion rather than the LLN criterion. The hypothesis that the incidence rate would be lower using the LLN criterion was confirmed. We also hypothesised that airflow limitation would be significantly associated with smoking category and age but not with sex. Compared to never-smokers, current smokers (but not ex-smokers) have significantly higher incidence rates, regardless of which criterion is applied. The analyses involving pack-years suggested that there might be an accumulative effect of smoking on the incidence rates in older age groups. There was a considerable age effect present for both criteria. However, relative to the fixed ratio criterion this effect was somewhat attenuated with LLN. There was a significant relationship between sex and airflow limitation for LLN. Thus the last part of the primary hypothesis regarding incidence was not confirmed for LLN. 
TABLE 4 Incidence rates and results from Poisson regression with airflow limitation defined as $r<0.7$

\begin{tabular}{|c|c|c|c|c|c|c|}
\hline Risk factors & $\begin{array}{c}\text { Mean } \\
\text { age }^{\#} \\
\text { years }\end{array}$ & Person-years & $\begin{array}{c}\text { Crude incidence per } \\
1000 \text { person-years }(95 \% \mathrm{CI})\end{array}$ & $\begin{array}{l}\text { Adjusted (LSM) incidence per } \\
1000 \text { person-years }(95 \% \mathrm{CI})^{\pi}\end{array}$ & $\begin{array}{l}\text { Crude } \\
\text { RR }\end{array}$ & $\begin{array}{l}\text { Adjusted RR } \\
(95 \% \mathrm{Cl})^{\pi}\end{array}$ \\
\hline Male & 69.0 & 2099 & $26.2(20.1-34.1)$ & $32.1(24.0-43.0)$ & 0.88 & $0.95(0.66-1.38)$ \\
\hline Female & 71.7 & 2547 & $29.8(23.8-37.4)$ & $33.8(26.4-43.2)$ & & \\
\hline \multicolumn{7}{|l|}{ Age group" years } \\
\hline $80-89$ & 83.2 & 889 & $50.6(37.8-67.8)$ & $57.5(41.8-79.2)$ & 2.84 & $3.15(2.05-4.86)$ \\
\hline $90-100$ & 90.7 & 138 & $43.6(19.6-97.1)$ & $51.8(23.0-116.8)$ & 2.45 & $2.84(1.19-6.76)$ \\
\hline \multicolumn{7}{|l|}{ Smoking category ${ }^{\#}$} \\
\hline Current smoker & 66.9 & 599 & $33.4(21.6-51.8)$ & $48.5(30.0-78.5)$ & 1.30 & 1.75 (1.04-2.93) \\
\hline Ex-smoker & 69.2 & 1728 & $29.5(22.4-38.8)$ & $37.6(26.8-52.6)$ & 1.15 & $1.36(0.91-2.02)$ \\
\hline Never-smoker & 72.4 & 2295 & 25.7 (19.9-33.2) & $27.8(20.5-37.8)$ & & \\
\hline
\end{tabular}

Data are presented as $n$, unless otherwise stated. LSM: least-squares means; RR: rate ratio. \#: smoking habits and age at baseline;

ף: covariates were sex, age group and smoking category.

\section{Consideration of possible mechanisms and explanations}

As expected, the incidence rate is consistently higher when using the fixed ratio criterion rather than the LLN criterion and there are effects of age and smoking. This would imply that in a geriatric population higher age and active smoking habits are independent risk factors for developing COPD even when LLN criteria are used.

A potential sex effect for LLN incidence has, at least partially, been observed previously for younger subjects [10], and previous studies have suggested that females may be more susceptible to tobacco smoke than males [16]. Other potential mechanisms behind the sex effect may include effects of sex hormones [27], susceptibility due to dimensional differences [28] and morphological differences suggesting a difference in the natural history of COPD [29]. The present study included only post-menopausal females and the results may not translate to younger females. The observed sex effect could theoretically be affected by higher awareness of COPD in male patients, leading to better treatment and spirometry performance. With few incident subjects, the observed effect may possibly be an artefact of natural variability, measurement error and dichotomising a continuous outcome to either obstructive or non-obstructive.

\section{Comparison of incidence rates with relevant findings from other published studies}

We found nine recent studies (published after the year 2000) that report spirometry-based incidence [2-10]. One of these only studied young adults and another only studied symptomatic subjects; they were therefore excluded from further comparison $[2,5]$.

\section{Previous studies using LLN criteria}

Little is known of the incidence of COPD among subjects aged $>80$ years considering that a fixed ratio criterion consistently overdiagnoses subjects aged $>50$ years [18]. Although there is controversy regarding the use of a fixed ratio (especially among elderly subjects), studies reporting LLN-based incidence rates are scarce. A Japanese study published in 2011 (including only male subjects $(n=913)$ ) reports incidence using both LLN and GOLD diagnostic criteria [9] (table 6). This study's results are not entirely comparable to ours since it uses a fixed ratio cut-off of 0.7 to exclude obstructive subjects at baseline for the LLN incidence calculations. The authors reported an overall incidence rate of 9.8 and 8.3 per 1000 person-years using LLN and GOLD criteria, respectively. The study's relatively young population (mean age 48 years) could explain the overall slightly higher incidence rate using LLN rather than the GOLD criterion. In summary, the 2011 Japanese study reported a significantly higher incidence rate among younger subjects and a lower incidence rate among older subjects using the LLN criterion compared to the GOLD criterion. A contributory factor to be considered was that active smokers were overrepresented among the younger subjects.

Another study of young adults (aged 20-44 years) with data from 10 European countries also reports incidence rate using two different versions of LLN and a fixed-ratio criterion [10]. In contrast to the Japanese study, this study reports lower incidence rate for LLN compared to the fixed-ratio analysis in 
TABLE 5 Incidence rates and results from Poisson regression with airflow limitation defined as $r<$ lower limit of normal

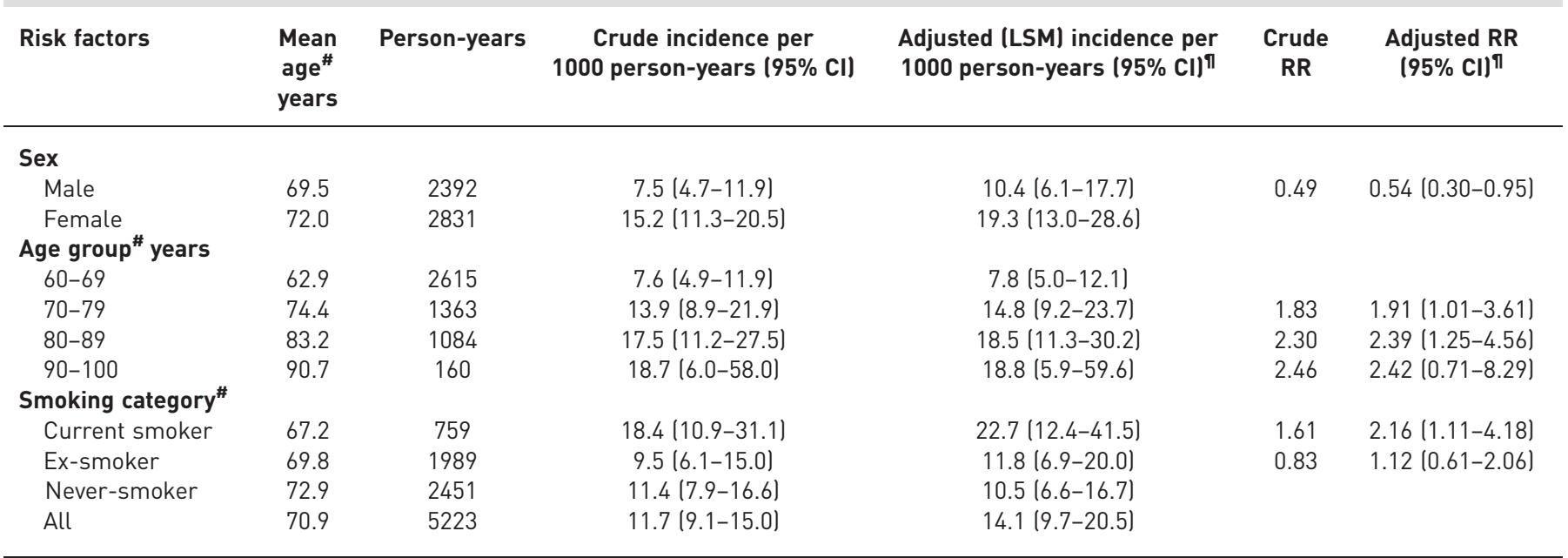

Data are presented as $n$, unless otherwise stated. LSM: least-squares means; RR: rate ratio. ${ }^{\#}$ : smoking habits and age at baseline; ${ }^{\text {: }}$ covariates were sex, age group and smoking category.

spite of the young study population. They also report higher incidence rate for females using the LLN criteria, but the results are not conclusive as they depend on which LLN criterion is applied.

Previous studies using GOLD criteria

We found five additional studies that report spirometry-based incidence rates in general populations using current GOLD criteria [2, 3, 6-8]. For easier comparison with our own results we calculated approximated overall incidence rate per 1000 person-years based on data published from four of these studies (table 6). The Danish study [7] published in 2006 does not provide sufficient information for comparison of overall incidence rate. The four remaining studies reported a wide spread of overall incidence rates ranging from 5.1 to 19.8 cases per 1000 person-years $[3,4,6-8]$. There seems to be a relationship between age of the study populations and reported incidence rates. There was no reported incidence rate for subjects aged $>80$ years in the previous studies for comparison. Since our study population has by far the highest average age, we are not surprised to find that our overall fixed ratio incidence rate was the highest reported. Another factor that might lead to higher incidence rates in our study is that, in an attempt to reduce selection bias, we included subjects who are too frail to visit the research centre. This was achieved by making house calls.

Incidence rates for current smokers were, 9.6, 26.9 and 11.0 for the Norwegian study [6], Swedish study [3] and 2007 Japanese study [4], respectively. The other studies did not report sufficient data for

FIGURE 2 The nonlinear effect of age as a continuous covariate on incident airflow limitation for lower limit of normal (LLN) and fixed-ratio criteria.

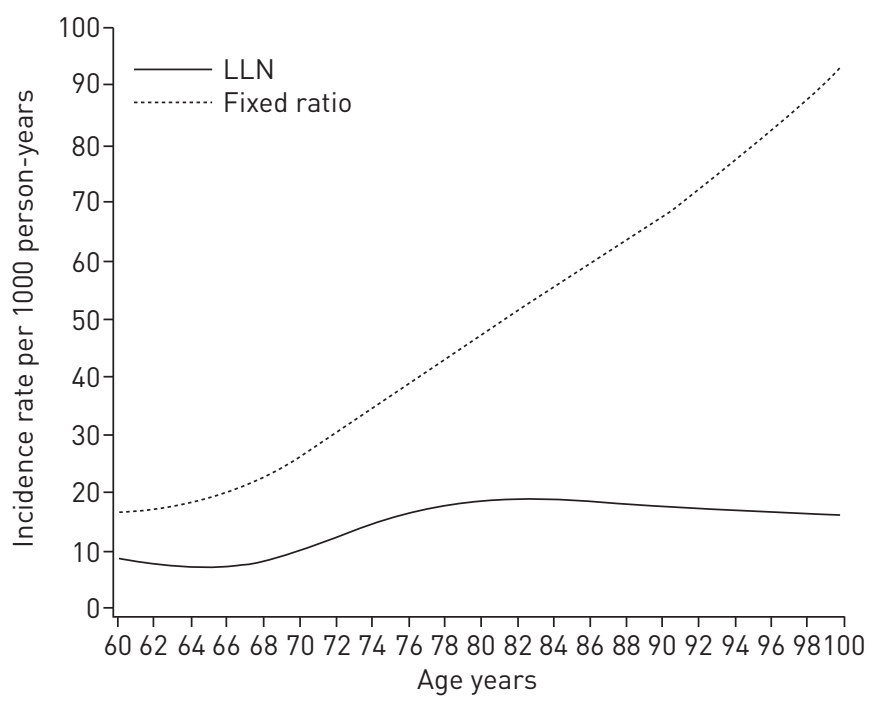


comparison. Again, the incidence rates were low in the Norwegian and Japanese studies compared to the older populations in the previous Swedish study and our cohort of current smokers.

\section{Comparison of smoking habits}

Our study population reports the lowest percentage of overall current smokers at baseline (13\%), compared to the previous studies where it ranged from $25 \%$ to $71 \%$ (table 6). In contrast, our percentage of former smokers was the highest reported (38\%), compared to the five previous studies. The other studies reported former smoker percentages between $18 \%$ and 35\%. Considering that our population's proportion of current smokers decreases with increasing age, it is likely that the inclusion of the oldest old explains why we found a relatively low percentage of current smokers and a high percentage of former smokers.

\section{Comparison of predictors}

We found that the incidence rate of airflow limitation was significantly higher for the 70-79- and 8089 -year-old cohorts compared to the youngest age group. This was also true for the 90-100-year-old cohort when using the fixed-ratio criterion but not when applying the LLN criterion. This was most likely a consequence of lack of power. Furthermore, we observed that current smokers have a significantly higher incidence rate than never-smokers. This is in accordance with previous studies (although only among males in the 2007 Japanese study) [3, 4, 6-8]. In accordance with our study the two previous studies that calculated dose-response relationships between estimated lifetime tobacco exposure and incidence found incidence and estimated tobacco exposure to be significantly correlated $[4,6]$. In accordance with all but three of the six previously mentioned, we did not find any significant relationship between incident airflow limitation based on a fixed ratio criterion and sex $[4,8,10]$. We did see a sex effect when using the LLN criterion. This result is partially supported by a previous study [10]. Being a former smoker did not significantly increase the incidence rate compared to never-smokers, regardless of which criterion was used. This was unexpected, as a previous study [10], the Norwegian study [6] and the 2007 Japanese study (although only for males) [4] which analysed smoking as a possible predictor of a fixed ratio-based incidence, reported contrary results. Possible reasons for this are discussed later.

\section{Limitations and weaknesses of the study}

We tried to reduce selection bias by making house calls. Still, participants were younger and were probably healthier than non-participants in the main study. Follow-up rates were lower for more severe stages of airflow limitation compared to mild stages. Subjects with two spirometries were likely to be fitter than those with baseline spirometry only, with the discrepancy being larger for older age groups. This may have resulted in generally lower incidences and weakened the age effect on incidence. It is likely that a fairly high proportion of subjects (in particular among those with poor pulmonary function at baseline) received treatment for airway obstruction between examinations. This may have led to both underestimation of incidence and a high proportion of incident negative subjects. In summary, it is likely that this study

TABLE 6 Approximated overall incident cases of airflow limitation/chronic obstructive pulmonary disease per 1000 person-years from previous studies on general populations using current Global Initiative for Chronic Obstructive Lung Disease (GOLD) and lower limit of normal (LLN) diagnostic criteria

\begin{tabular}{|c|c|c|c|c|c|c|c|}
\hline \multirow[t]{2}{*}{ First author [ref.] } & \multirow[t]{2}{*}{$\begin{array}{l}\text { At-risk } \\
\text { subjects }\end{array}$} & \multirow[t]{2}{*}{ Country } & \multirow{2}{*}{$\begin{array}{l}\text { Mean } \\
\text { age } \\
\text { years }\end{array}$} & \multirow{2}{*}{$\begin{array}{c}\text { Current } \\
\text { smokers } \\
\%\end{array}$} & \multirow{2}{*}{$\begin{array}{c}\text { Male/ } \\
\text { female } \\
\% / \%\end{array}$} & \multicolumn{2}{|c|}{$\begin{array}{l}\text { Incidence } \\
\text { rate }\end{array}$} \\
\hline & & & & & & GOLD & LLN \\
\hline JoHANNESSEN [6] & 869 & Norway & 41 & 27 & $53 / 47$ & 5.1 & $\#$ \\
\hline KоJIMA [4] & 17106 & Japan & 48 & 30 & $65 / 35$ & 6.3 & $\#$ \\
\hline OMORI [9] & 913 & Japan & 48 & 71 & $100 / 0$ & 8.3 & 9.8 \\
\hline LiNDBERG [3] & 963 & Sweden & ๆ & 25 & $49 / 51$ & 15.7 & \# \\
\hline Vestbo [8] & 12309 & Denmark & 52 & 61 & $\#$ & $19.8^{+}$ & $\#$ \\
\hline DE MARCo [10] & 4425 & Multinational & $\S$ & $\#$ & $\#$ & 2.88 & $1.85 / 2.41$ \\
\hline \multicolumn{8}{|l|}{ Present study } \\
\hline LLN & 892 & Sweden & 71 & 14 & $46 / 54$ & & 11.7 \\
\hline Fixed ratio & 795 & Sweden & 70 & 13 & $45 / 55$ & 28.2 & \\
\hline
\end{tabular}

Data are presented as $n$, unless otherwise stated. " : data not available; " : data not available, age at study entry 46-77 years; ${ }^{+}$: calculated from 5-year follow-up; ${ }^{\S}$ : data not available, age at study entry $20-44$ years. 
underestimates the true incidence of airflow limitation in the underlying population as a result of selection of the fittest.

Failure to perform an acceptable spirometry at baseline resulted in the loss of a rather young subpopulation with a high percentage of smokers. It is plausible that this loss was to a large extent due to technical problems. This could have affected the results. However, the baseline characteristics did not differ substantially between the 2025 subjects with spirometry and the 2336 subjects examined. Furthermore, pulmonary function at follow-up is not different in the lost group compared to the 984 subjects with two measurements. We expect this to be mainly an issue of loss of power.

A bronchodilator was not administered at baseline. It is likely that subjects close to the cut-off value of a fully reversible versus not fully reversible airway obstruction were wrongfully excluded from the analysis. This may have led to an underestimation of the incidence, since these subjects were likely to have had an increased risk of developing post-bronchodilator airflow limitation. The sensitivity analyses we conducted also point to relatively modest underestimations of the incidence rates with little effect on predictors.

Due to lack of alternative equations for subjects aged $>95$ years we extrapolated the spirometric prediction equations for individuals aged 25-95 years for a handful of subjects aged $>95$ years at follow-up.

Former smokers did not differ significantly from never-smokers regarding incidence, unlike findings in previous studies $[6,7]$. This may be explained by the inability to accurately measure exposure, which results in a bias towards unity. This effect has been described before as regression dilution bias [30]. It is likely that the somewhat crude predictors of smoking category and pack-years are affected by regression dilution bias. The pack-years predictor is also likely to be affected by recall bias.

Variables such as occupational exposure [31,32] and socioeconomic status [33, 34] that have previously been linked to COPD were not included. Air pollution has also previously been suggested as a predictor of COPD; however, a recently published review [35] concluded that the evidence was not conclusive. Additionally, our study includes randomised participants from both urban and rural areas, which should reduce the effects of any differences in air pollution between rural and urban areas.

\section{Possible contributions of the study}

Since this is one of the first published studies reporting LLN-based incidence data and the first to use the GLI-2012 reference equations, we hope that this study will contribute to the current knowledge of the epidemiology of COPD. The inclusion of the oldest old, with data from 100-year-old subjects at follow-up, further adds to the usefulness of this study. The evidence for using the fixed-ratio criterion when diagnosing airflow limitation in the very old was found to be weak. In fixed-ratio studies the one predictor that is most correlated to incidence is age, partly as a result of misclassification, with high numbers of false positives in high age groups. This study describes the incidence of airflow limitation using modern age-dependent reference equations with the hope of coming closer to the true underlying incidence of COPD. We found that female sex may be a risk factor for developing airflow limitation and consequently COPD. This finding has to be corroborated.

\section{Acknowledgements}

The authors thank Helen Legrand (Department of Health Sciences, Division of Geriatric Medicine, Scania University Hospital, Lund University, Malmö, Sweden) for her assistance with language.

\section{References}

1 Rycroft C, Heyes A, Lanza L, et al. Epidemiology of chronic obstructive pulmonary disease: a literature review. Int J Chron Obstruct Pulmon Dis 2012; 7: 457.

2 Lindberg A, Jonsson A-C, Rönmark E, et al. Ten-year cumulative incidence of COPD and risk factors for incident disease in a symptomatic cohort. Chest 2005; 127: 1544-1552.

3 Lindberg A, Eriksson B, Larsson LG, et al. Seven-year cumulative incidence of COPD in an age-stratified general population sample. Chest 2006; 129: 879-885.

4 Kojima S, Sakakibara H, Motani S, et al. Incidence of chronic obstructive pulmonary disease, and the relationship between age and smoking in a Japanese population. J Epidemiol 2007; 17: 54-60.

5 de Marco R, Accordini S, Cerveri I, et al. Incidence of chronic obstructive pulmonary disease in a cohort of young adults according to the presence of chronic cough and phlegm. Am J Respir Crit Care Med 2007; 175: 32-39.

6 Johannessen A, Omenaas E, Bakke P, et al. Incidence of GOLD-defined chronic obstructive pulmonary disease in a general adult population. Int J Tuberc Lung Dis 2005; 9: 926-932.

7 Løkke A, Lange P, Scharling H, et al. Developing COPD: a 25 year follow up study of the general population. Thorax 2006; 61: 935-939.

8 Vestbo J, Lange P. Can GOLD Stage 0 provide information of prognostic value in chronic obstructive pulmonary disease? Am J Respir Crit Care Med 2002; 166: 329-332.

9 Omori H, Nagano M, Funakoshi Y, et al. Twelve-year cumulative incidence of airflow obstruction among Japanese males. Intern Med 2011; 50: 1537-1544. 

cohort of young adults. Am J Respir Crit Care Med 2011; 183: 891-897.

11 Mohamed Hoesein FAA, Zanen P, Lammers J-WJ. Lower limit of normal or FEV1/FVC $<0.70$ in diagnosing COPD: an evidence-based review. Respir Med 2011; 105: 907-915.

12 Medbø A, Melbye H. Lung function testing in the elderly - can we still use $\mathrm{FEV}_{1} / \mathrm{FVC}<70 \%$ as a criterion of COPD? Respir Med 2007; 101: 1097-1105.

13 Swanney MP, Ruppel G, Enright PL, et al. Using the lower limit of normal for the FEV1/FVC ratio reduces the misclassification of airway obstruction. Thorax 2008; 63: 1046-1051.

14 Hardie JA, Buist AS, Vollmer WM, et al. Risk of over-diagnosis of COPD in asymptomatic elderly never-smokers. Eur Respir J 2002; 20: 1117-1122.

15 Wollmer P, Engström G. Fixed ratio or lower limit of normal as cut-off value for FEV1/VC: an outcome study. Respir Med 2013; 107: 1460-1462.

16 Global Initiative for Chronic Obstructive Lung Disease (GOLD). Global Strategy for the Diagnosis, Management and Prevention of COPD. www.goldcopd.org Date last accessed: June 15, 2015. Date last updated: January 2015.

17 Pellegrino R, Viegi G, Brusasco V, et al. Interpretative strategies for lung function tests. Eur Respir J 2005; 26: 948-968.

18 Quanjer PH, Stanojevic S, Cole TJ, et al. Multi-ethnic reference values for spirometry for the 3-95-yr age range: the global lung function 2012 equations. Eur Respir J 2012; 40: 1324-1343.

19 Stenhagen M, Ekström H, Nordell E, et al. Falls in the general elderly population: a 3- and 6-year prospective study of risk factors using data from the longitudinal population study "Good ageing in Skane". BMC Geriatr 2013; 13: 81 .

20 Ekström H, Elmståhl S. Pain and fractures are independently related to lower walking speed and grip strength: results from the population study "Good Ageing in Skåne”. Acta Orthop 2006; 77: 902-911.

21 Lagergren M, Fratiglioni L, Hallberg IR, et al. A longitudinal study integrating population, care and social services data. The Swedish National study on Aging and Care (SNAC). Aging Clin Exp Res 2004; 16: 158-168.

22 Standardization of Spirometry, 1994 Update. American Thoracic Society. Am J Respir Crit Care Med 1995; 152: 1107-1136.

23 Quanjer PH, Stanojevic S, Cole TJ, et al. Implementing GLI-2012 regression equations. Global Lung Function Initiative. www.ers-education.org/media/media.aspx?idmedia=266709 Date last accessed: June 15, 2015.

24 Kainu A, Lindqvist A, Sarna S, et al. FEV1 response to bronchodilation in an adult urban population. Chest 2008; 134: 387-393.

25 Turkeshi E, Vaes B, Andreeva E, et al. Airflow limitation by the Global Lungs Initiative equations in a cohort of very old adults. Eur Respir J 2015; 46: 123-132.

26 Vaz Fragoso CA, Concato J, McAvay G, et al. The ratio of FEV 1 to FVC as a basis for establishing chronic obstructive pulmonary disease. Am J Respir Crit Care Med 2010; 181: 446-451.

27 Becklake MR, Kauffmann F. Gender differences in airway behaviour over the human life span. Thorax 1999; 54: 1119-1138.

28 Kanner RE, Connett JE, Altose MD, et al. Gender difference in airway hyperresponsiveness in smokers with mild COPD. The Lung Health Study. Am J Respir Crit Care Med 1994; 150: 956-961.

29 Dransfield MT, Washko GR, Foreman MG, et al. Gender differences in the severity of CT emphysema in COPD. Chest 2007; 132: 464-470.

30 MacMahon S, Peto R, Cutler J, et al. Blood pressure, stroke, and coronary heart disease. Part 1, Prolonged differences in blood pressure: prospective observational studies corrected for the regression dilution bias. Lancet 1990; 335: 765-774

31 Pauwels RA, Rabe KF. Burden and clinical features of chronic obstructive pulmonary disease (COPD). Lancet 2004; 364: 613-620.

32 Bergdahl IA, Torén K, Eriksson K, et al. Increased mortality in COPD among construction workers exposed to inorganic dust. Eur Respir J 2004; 23: 402-406.

33 Bakke PS, Hanoa R, Gulsvik A. Educational level and obstructive lung disease given smoking habits and occupational airborne exposure: a Norwegian community study. Am J Epidemiol 1995; 141: 1080-1088.

34 Prescott E, Vestbo J. Socioeconomic status and chronic obstructive pulmonary disease. Thorax 1999; 54: 737-741.

35 Schikowski T, Mills IC, Anderson HR, et al. Ambient air pollution: a cause of COPD? Eur Respir J 2014; 43: 250-263. 